\title{
Contrassensos da Rede de Atenção Psicossocial Direcionada ao Curso do Adolescente
}

\author{
Usuário de Crack
}

\author{
Contradictions of the Psychosocial Care Network Oriented Towards the Care of \\ Teenage Crack Users \\ Juliane Portella Ribeiro ${ }^{1 *}$ Giovana Calcagno Gomes ${ }^{2 *}$ Adriane Domingues Eslabão ${ }^{3}$ * Priscila \\ Arruda da Silva ${ }^{4}$
}

\begin{abstract}
RESUMO
Objetivo: identificar os contrassensos da Rede de Atenção Psicossocial direcionada ao cuidado do adolescente usuário de crack. Métodos: Estudo exploratório e descritivo com abordagem qualitativa, realizado com 20 profissionais atuantes em um Centro de Atenção Psicossocial Álcool e outras Drogas. A coleta de dados ocorreu no primeiro semestre de 2017, por meio de entrevistas semiestruturadas. Os dados foram organizados e tratados pelo software Nvivo 11 e submetidos a Análise Temática. Resultados: Os contrassensos na dinâmica de funcionamento da rede envolvem a (Des)articulação da Rede de Atenção Psicossocial, o (Des)conhecimento dos usuários e profissionais acerca da Rede de Atenção Psicossocial e os (Des)ajustes envolvidos na assistência ao adolescente usuário de crack. Conclusões: os movimentos de construção desta rede precisam ser fortalecidos a partir da articulação de ações de prevenção ao uso de drogas, de tratamento e de reinserção social que utilizem os recursos existentes no território da própria comunidade do adolescente.
\end{abstract}

Palavras-chave: Adolescente. Usuários de Drogas. Avaliação em Saúde. Assistência Integral à Saúde.

\begin{abstract}
Objective: to identify the contradictions of the Psychosocial Care Network oriented towards the care of teenage crack users. Methods: exploratory and descriptive study, with a qualitative approach, performed with 20 professionals working in a Psychosocial Care Center for Alcohol and Other Drugs. Data collection occurred in the first half of 2017, through semi-structured interviews. Data were organized and treated by the software Nvivo 11, and then submitted to Thematic Analysis. Results: the contradictions in the dynamics of network operation involve the (dis)articulation of the Psychosocial Care Network, the (Un)knowledge of the users and professionals about the Psychosocial Care Network, and the (Mis)adjustments involved in the assistance to the adolescent crack user. Conclusions: the movements for constructing this network should to be strengthened from the articulation of preventive actions related to drug consumption, treatment, and social reintegration, using the resources existing in the territory of the adolescent's community.
\end{abstract}

Keywords: Adolescent. Drug Users. Health Evaluation. Comprehensive Health Care.

${ }^{1}$ Universidade Federal de Pelotas (UFPEL), Faculdade de Enfermagem. Pelotas, RS, Brasil. E-mail: ju_ribeiro1985@hotmail.com Correspondência: Rua Gomes Carneiro №1, 20 andar. Faculdade de Enfermagem, Universidade Federal de Pelotas. CEP: 96010-610 - Pelotas, RS, Brasil.

${ }^{2}$ Universidade Federal do Rio Grande (FURG), Escola de Enfermagem. Rio Grande, RS, Brasil. E-mail: giovanacalcagno@furg.br

${ }^{3}$ Universidade Federal do Rio Grande do Sul (UFRGS), Programa de Pós-Graduação da Escola de Enfermagem. Porto Alegre, RS, Brasil. E-mail: adrianeeslabao@hotmail.com

${ }^{4}$ Universidade Federal do Rio Grande (FURG), Escola de Enfermagem. Rio Grande, RS, Brasil. E-mail: patitaarruda@yahoo.com.br 


\section{INTRODUÇÃO}

A Organização Mundial de Saúde aponta que as pessoas com problemas de saúde mental relacionados ao consumo de álcool e outras drogas, e apesar de ser um grupo vulnerável, não têm sido consideradas pelas agendas de trabalho $\mathrm{e}$ desenvolvimento dos países. O que gera grande impacto econômico para as famílias e a sociedade, além da exclusão social, descriminação e estigmação ${ }^{1}$.

No Brasil as pesquisas apontam a grande vulnerabilidade em que vivem as pessoas em sofrimento psíquico pelo uso de drogas como o crack, visto que estes iniciam o seu uso na adolescência, são de classe social baixa, filhos de pais separados e que permanecem sobre os cuidados da mãe e morando com os irmãos, com baixa escolaridade, desempregados, com a perda importante de alguém da família e que já pensaram em suicídio ${ }^{2-3}$.

No entanto, tais dados em muitos momentos passam longe da compreensão das estratégias de cuidado e das políticas de planejamento social. Agravando essa situação, muitos usuários de álcool, crack e outras drogas, e seus familiares sofrem com o preconceito e estigma, o que dificulta o seu acesso e vinculo aos serviços de cuidado ${ }^{4}$.

Por essa razão, o sistema de saúde tem se mobilizado no sentido de instituir uma Rede de Atenção Psicossocial (RAPS) com serviços substitutivos aos hospitais psiquiátricos. Assim, o cuidado deve iniciar no território com medidas de prevenção e promoção à saúde se estendendo a uma rede de cuidado 5 . Com a RAPS há a possibilidade de criação, ampliação e articulação de pontos de atenção à saúde para os adolescentes com necessidades decorrentes do uso de crack, pensando diversas estratégias possíveis de suporte por meio da integração de pontos de atenção.

Para os casos de consumo abusivo de drogas, existe na rede os seguintes serviços: Unidades Básicas de Saúde com ou sem a Estratégia de Saúde da Família (ESF), os Centros de Atenção Psicossocial
Álcool e outras Drogas (CAPS AD), as Unidades de Acolhimento Infantojuvenil e as Unidades de Acolhimento Adulto, Equipes de Consultório na Rua e de Redução de Danos e os leitos psiquiátricos em unidades de hospital geral e em hospitais psiquiátrico, pois esta estrutura permanece na rede e é muito utilizada mesmo com uma grande luta pela sua substituição ${ }^{5}$.

No entanto, é notória a lacuna existente na RAPS quanto ao cuidado do adolescente usuário de drogas. Tal entendimento se sustenta no fato de que ainda é incipiente a instalação de serviços especializados a esta população, tanto que o Centro de Atenção Psicossocial Infantil (CAPSi) como o CAPS AD atendem esse público, o que, consequentemente, favorece a fragmentação e a desresponsabilização pela assistência e pelo cuidado ${ }^{6}$.

Além disso, observa-se ainda o emprego de medidas higienistas de limpeza das ruas, principalmente em grandes áreas urbanas e turísticas. Medidas como prisão, recolhimento involuntário temporário, e recolhimento compulsório de crianças que ficam sobre a responsabilidade de conselheiros tutelares ${ }^{7}$.

Diante do exposto, o presente estudo tem por objetivo identificar os contrassensos da Rede de Atenção Psicossocial (RAPS) direcionada ao cuidado do adolescente usuário de crack, a partir da perspectiva dos profissionais atuantes no Centro de Atenção Psicossocial em Álcool e outras Drogas (CAPS AD).

\section{MÉTODO}

Trata-se de um estudo descritivo e exploratório com abordagem qualitativa dos dados, vinculado a um projeto de pesquisa amplo, intitulado "(Des)caminhos percorridos pelo adolescente usuário de crack na rede de atenção psicossocial: contribuição para a Enfermagem", desenvolvido em um município de médio porte do interior do Rio Grande do Sul que integra o Programa "Crack, é possível vencer".

O Centro de Atenção Psicossocial Álcool e 


\section{ARTIGO ORIGINAL}

Drogas (CAPS AD III) foi elegido como local de coleta de dados, uma vez que possui especificidades distintas da pluralidade dos demais serviços que compõem a Rede de Atenção Psicossocial do Município. A maioria dos serviços atua de forma pontual e delimitada por tempo específico, enquanto que o CAPS AD III é um serviço de atenção integral e continuada às pessoas com necessidades em decorrência do uso de álcool, crack e outras drogas. Além disso, na realidade da RAPS do município em questão, os casos de adolescentes usuários de drogas direcionados e acompanhados pelo CAPS infantil é ínfimo, quando não é nulo.

Participaram do estudo 20 profissionais atuantes no CAPS AD. A seleção dos participantes foi intencional, de acordo com os seguintes critérios de inclusão: ser trabalhador de nível médio ou superior que compõem a equipe multiprofissional do serviço e possuir no mínimo seis meses de atuação no serviço. Foram excluídos profissionais em férias ou licença saúde no período de coleta dos dados.

O número de participantes foi definido pela saturação dos dados definida quando, na avaliação do pesquisador, ocorre uma certa redundância ou repetição, não sendo considerado relevante persistir na coleta de dados. A coleta de dados ocorreu no primeiro semestre de 2017, por meio de entrevistas semiestruturadas realizadas por um único entrevistador.

Com vistas à privacidade dos participantes, as entrevistas foram realizadas em uma sala do serviço, respeitando a disponibilidade e funcionamento do CAPS AD. Para preservar o conteúdo original e aumentar a acurácia dos dados obtidos, as entrevistas foram capturadas por um gravador de áudio e, posteriormente, transcritas na íntegra.

Para a organização e tratamento dos dados, empregou-se o software Nvivo 11, programa que auxilia na análise de material qualitativo com ferramentas de codificação e armazenamento de textos $^{8}$. Posteriormente, foram analisados e categorizados conforme a Análise Temática. Utilizou-se a ferramenta da nuvem de palavras, criada a partir do software para visualizar a representatividade e ocorrência das mesmas em uma dada categoria, uma vez que esta técnica emprega diferentes tamanhos e fontes de letras para enfatizar a frequência da palavra no texto analisado.

Com vistas a garantir os princípios éticos relativos à pesquisas que envolvem seres humanos, os participantes foram incluídos no estudo somente após manifestarem sua concordância em participar do mesmo através da assinatura do Termo de Consentimento Livre e Esclarecido (TCLE). Além disso, o anonimato dos profissionais do CAPS Ad participantes do estudo foi preservado por meio do emprego da letra $\mathrm{P}$, sucedidas do número da entrevista.

Deste modo, os preceitos éticos da realização de pesquisa envolvendo seres humanos foram garantidos, conforme a Resolução no 466 , de 12 de dezembro de 2012. O projeto foi encaminhado ao Comitê de Ética em Pesquisa e aprovado mediante o Certificado de Apresentação para Apreciação Ética (CAEE) no 60649016.9.0000.5324, Parecer no 4/2017.

\section{RESULTADOS E DISCUSSÃO}

A partir da análise dos dados, a dinâmica de funcionamento da rede de atenção psicossocial direcionada ao cuidado do adolescente usuário de crack pode ser compreendida pelas categorias: "(Des)articulação da Rede de Atenção Psicossocial", "(Des)conhecimento dos usuários e profissionais acerca da Rede de Atenção Psicossocial" e "(Des)ajustes envolvidos na assistência ao adolescente usuário de crack.

\section{(Des)articulação da Rede de Atenção Psicossocial}

A RAPS foi estruturada para o cuidado ao adolescente em uso de crack, no município, mas ainda é insipiente, havendo um consenso da necessidade de movimentos de aproximação entre os serviços da atenção especializada e os demais serviços pelos quais ele transita, como CAPSi, 


\section{ARTIGO ORIGINAL}

escolas, serviços de atenção primária, Unidades Básicas de Saúde (UBS) e ESF, Conselho Tutelar, CRAS, promotoria e SAMU, na tentativa de constituir uma rede intersetorial, articulando ações no território (Figura I).

Esse ano a gente está se aproximando mais do CAPSi, pensando em rede, mas ainda está muito insípida, assim como com a atenção básica. A gente está pensando em se organizar para crescer essa rede, fazer reuniões coletivas, tem que juntar o CAPSi, o Conselho Tutelar, a promotoria, o CAPS $A D$. A gente tem que conversar mais, tem algumas coisas meio atravessadas (p7).

Acredito que há necessidade de maior diálogo entre os dispositivos, maior envolvimento, principalmente com o CAPSi, pois muitas crianças são tratadas no CAPSi e quando passam a utilizar entorpecentes, muitas vezes, se quebra esse vínculo e automaticamente eles passam a ser usuários do CAPS AD (p5).

Eu acho que a principal [dificuldade] é a articulação da rede. Que pudesse estar tudo conectado, que não tivéssemos tanta dificuldade de acesso em outros serviços, porque esses adolescentes que estão sendo acompanhados aqui, eles estão em uma área de maior vulnerabilidade, $e$ são locais que tem estratégia de saúde da família. Então, que a gente pudesse ter mais parceiros nesse território, porque às vezes é uma frustração, sabe? A gente não dá conta ( $p 15)$.

Eu acho que discutir essa problemática $e$ trabalhar um pouco mais integrada com a rede como um todo, desde a rede básica que conhece a família, como CRAS, com toda a rede. Que a gente possa traçar juntamente com os demais profissionais um plano para esse adolescente, não só um plano como atividade do CAPS, mas um plano geral, em todos os sentidos (p11).

Acho que tem que ser muito além, porque só o CAPS e a unidade de acolhimento infantil jamais vão dar conta de toda demanda, tem que ser um trabalho intersetorial (p8).

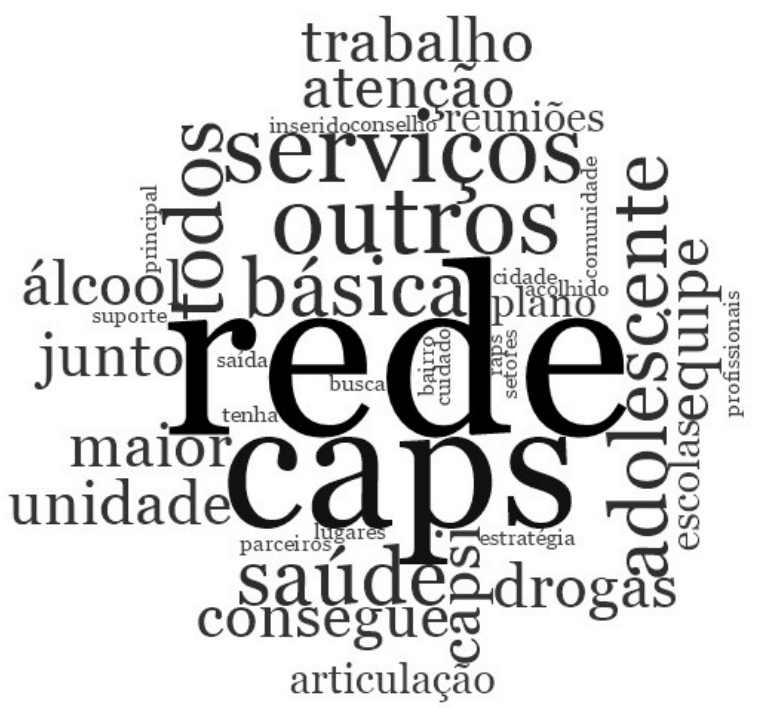

Figura 1 - Nuvem de palavras por frequência da codificação da categoria "(Des)articulação da RAPS", via QSR NVivo. Pelotas/RS, 2017.

O modo de Atenção Psicossocial que busca superar o modelo hospitalocêntrico de cuidado é de base territorial, portanto, deve funcionar como elemento integrado com o território, atuando através de ferramentas focadas na liberdade, autonomia, e busca da interrupção de internações frequentes. Para tanto, posiciona os CAPS como serviços que ocupam função estratégica na missão de substituição da lógica manicomial, articulando o trabalho em rede ${ }^{9}$.

Nesta perspectiva de cuidado, o CAPS faz parte da Rede de Atenção Psicossocial e deve se articular a uma rede maior, intersetorial, para realizar práticas de cuidado na integralidade das necessidades de cada pessoa e família. Para operar nessa lógica depende-se das condições que o CAPS possui de se articular, de produzir trabalho compartilhado diante de uma nova forma de olhar para a rede, pautada na produção de encontros cada vez mais singulares entre o equipamento e os sujeitos alvos do cuidado ${ }^{10}$.

Os trabalhadores do CAPS AD III reconhecem a importância destes movimentos em rede, da necessidade de articular e compartilhar o cuidado, do valor que possui o território enquanto espaço 
de vida, de subjetividade, de trocas, de prevenção e reinserção social. Entretanto, os movimentos em construção desta rede são tímidos e precisam ser fortalecidos, pois apenas deste modo será possível um cuidado ampliado em saúde mental.

Nesta perspectiva, a construção de uma rede intersetorial com articulação de ações no território é explicitada como forma de atuar na prevenção ao uso de drogas e no tratamento e na reinserção social pós alta do CAPS AD III, por meio de estratégias que utilizem os recursos da própria comunidade.

Penso que o atendimento, o cuidado, deverá ter articulação maior com as escolas, entidades de bairros iniciando ainda antes da adolescência, ou seja, é necessário investir mais em prevenção primária (p12).

Eu penso que seria essencial ir para comunidade, estar na comunidade. Aqui o serviço social até busca para eles não ficarem inseridos só aqui. A gente não quer ele institucionalizado, a gente quer que ele vá para vida de novo, que ele consiga se controlar. E, se ele não consegue deixar de fazer o uso, que esse uso seja mais controlado, recreativo. Já que a gente sabe que a abstinência nem todos conseguem. Então, o objetivo é fazer uma redução, que eles consigam conviver com essas coisas todas, mas não deixem de viver por isso (p19).

Para o adolescente acolhido hoje já temos que dar atenção para a saída dele do CAPS. E para planejar essa saída eu já tenho que estar convocando todos esses setores e profissionais que poderão fazer o suporte. Se não ele vai sair daqui ele vai voltar ( $p 8)$.

Pesquisadores apontam que a existência de um fórum intersetorial para a discussão entre profissionais pode ser considerado como potente tendo em vista a complexidade do cuidado ${ }^{11}$. No entanto, a literatura científica constantemente mostra a desarticulação entre os serviços intersetoriais e em alguns casos entre os serviços de uma mesma rede ${ }^{11-15}$.

Corroborando com os resultados do presente estudo, pesquisadores apontam que um dos maiores desafios identificados por trabalhadores que atuam no CAPS AD é a dificuldade de um trabalho em rede que ocorra de forma realmente efetiva, corresponsável, cujas ações em saúde possam ser pensadas em conjunto, propondo o fortalecimento das estratégias para o enfrentamento do uso de álcool e outras drogas, envolvendo os CAPS, os profissionais, e a rede de atenção em saúde ${ }^{15}$.

Embora o CAPS possa ser considerado um serviço resolutivo no sentido de evitar internações, desenvolver estratégias de controle da fissura, cuidado a medicação, e desenvolver redes de sistemas existentes no território, ainda há necessidade de avançar em ações em rede voltadas para o contexto social, cultural e histórico dos usuários. Deste modo, pela percepção das reais necessidades do usuário, pelo interesse em conhecê-lo e contextualizar a utilização da droga, constata-se que por meio de uma clínica ampliada do sujeito torna-se possível criar novos contornos do trabalho em rede, incluindo espaços de cuidado que reconheçam o território ${ }^{16}$.

Embora a articulação entre os serviços seja necessária, existe ainda uma dificuldade em superar o estigma relacionado ao uso de drogas. Muitas vezes, isso implica na forma como o adolescente é acolhido e compreendido em suas necessidades. Não raro, o usuário de drogas é ignorado em sua completude, sendo percebido somente em relação à droga de uso.

Aqui na cidade, não são só aqui como em outros lugares que eu trabalhei, falou que usou alguma substância, vem para o AD. Então, eu acho que tem que ter muito cuidado com isso ( $p 7)$.

Os outros CAPS têm: - Ah, bebeu um pouquinho, vai procurar o CAPS AD. As vezes, é uma pequena recaída que pode ser manejada pelo próprio CAPS, mas se tem essa cultura de único caminho (p2).

A centralidade do cuidado dos usuários de crack e outras drogas no CAPS AD pode indicar importantes desafios, como: a fragmentação da 


\section{ARTIGO ORIGINAL}

atenção que fica focada no interior do CAPS AD, a falta de capacitação para atuar com essas demandas na atenção básica e em outros dispositivos de cuidado e o desconhecimento do papel dos CAPS na atenção ao sofrimento psíquico.

Pesquisa que analisou a articulação da rede no cuidado ao usuário de crack em um município da região metropolitana de Porto Alegre-RS, identificou a existência de uma compreensão distorcida da rede, em que entende-se que todos os problemas de saúde mental e o uso de drogas devem ser acolhidos e cuidados em serviços especializados e não em uma rede intersetorial ${ }^{11}$, fato que condiz com os resultados da presente pesquisa.

Deste modo, a escassa e demorada articulação em rede e a falta de compreensão e capacitação de alguns profissionais torna o cuidado ineficaz e com importantes desafios para superar práticas retrógradas e estigmatizantes ${ }^{12}$. É preciso investir em modos de cuidado que promovam encontros produtivos. Para tanto, é necessário maior investimento em educação permanente, pois o usuário de crack e outras drogas possui muitas demandas e não pode ser visto de modo fragmentado utilizando apenas um serviço da rede de atenção "o CAPS AD".

Isso traz à tona a discussão sobre o cuidado integral, um dos princípios do SUS, que não poderá ser alcançado apenas por um serviço ou profissional, mas por meio de uma rede de atenção. Por esta razão, questiona-se a composição dos cenários de cuidado enquanto lugares que fragmentam partes distintas do sofrimento humano, como o uso abusivo de álcool e outras drogas, de forma a potencializar soluções e/ou arranjos que acolham a pessoa de maneira integral e não somente o seu problema ${ }^{10}$.

Ademais, é importante lembrar que os CAPS $A D$ devem ficar responsáveis somente pelos casos mais graves e complexos identificados na comunidade, ou seja, aqueles casos que apresentam um padrão de dependência ou grave comprometimento sócio familiar, e não por toda e qualquer pessoa que tenha problemas com álcool e outras drogas. Essas pessoas, que constituem a maior parcela da população, podem e devem ser atendidas nos serviços de atenção básica de saúde, contando com o apoio dos CAPS AD, e devem atuar de forma articulada com o restante da rede de saúde e dos outros serviços necessários ao cuidado ${ }^{17}$.

\section{(Des)conhecimento da RAPS}

O relato dos participantes evidenciou que para melhorar o acesso do usuário e a articulação entre os serviços que compõem a RAPS, faz-se necessário trabalhar na divulgação e visibilidade da mesma para a população, mas principalmente para os serviços que atuam na comunidade. Os profissionais expõem o desconhecimento da população em relação à existência de um serviço especializado em dependência química no município, o que não raramente faz com que o serviço procurado e utilizado seja o hospital psiquiátrico (Figura II).

A população não tem conhecimento de como funciona o serviço de rede mental. Acho que a população em geral não tem muita noção de que tem um CAPS especializado em dependência química (p14).

Acho que ainda tem muita gente que não conhece, falta de conhecimento, informação, como chegar aqui, quais são os meios, porque a demanda pode ser espontânea, não precisa ter encaminhamento (p19).

Eu acredito que eles venham direto, quando eles sabem, porque tem muita gente na cidade que não sabe que existe um CAPS AD (p15).

A gente tem o mapeamento de todos os adolescentes que internaram e reinternaram no hospital psiquiátrico. Ano passado, mais da metade deles nunca tinham ido nem no CAPS $A D$ nem no CAPS i. Então, a gente foi atrás desses adolescentes para oferecer o serviço, para apresentar, para dizer que existe, para tentar acolhê-los no nosso serviço (p18). 


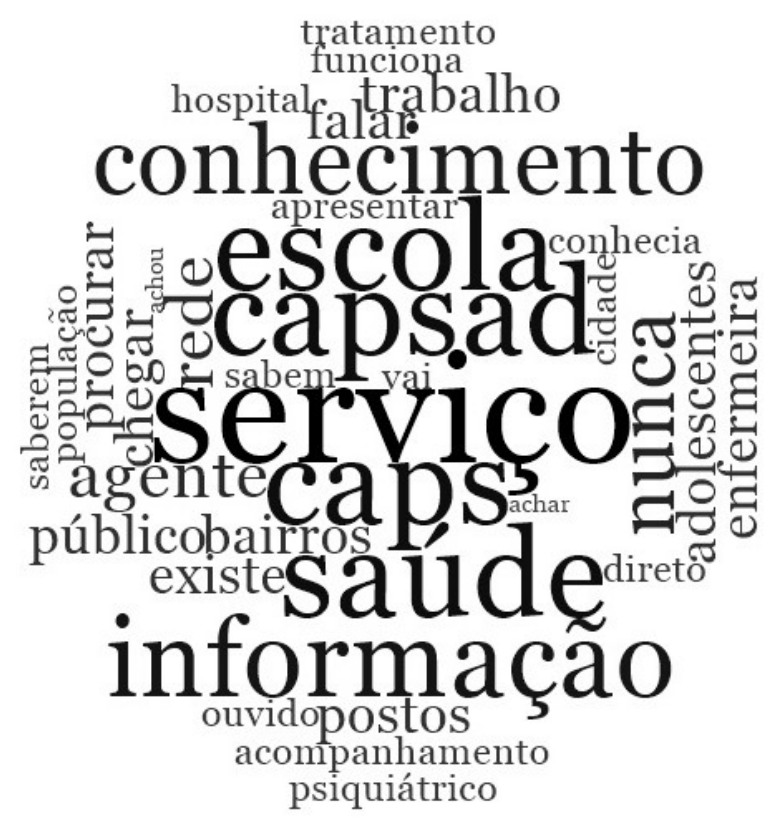

Figura 2 - Nuvem de palavras por frequência da codificação da categoria "(Des)conhecimento da RAPS", via QSR NVivo. Pelotas/RS, 2017.

O desconhecimento da rede é um ponto negativo, visto que não há como cuidar sem conhece-la, sem articulação e sem a discussão dos casos. Neste sentido, é preciso destacar o importante trabalho realizado por essa equipe ao mapear os usuários acessados pelo hospital psiquiátrico do município, buscando meios reais de minimizar os problemas gerados pelo desconhecimento da rede e uma forma de garantir e facilitar o acesso dos usuários ao CAPS AD.

$O$ desconhecimento da rede também foi identificado em outras pesquisas, a qual descreveu as características da articulação entre os serviços que compõem a rede de saúde mental infantojuvenil, através do sistema de referência e contrarreferência. Essa evidenciou que as dificuldades em dar respostas às necessidades do usuário estão relacionadas com a falta de comunicação e o desconhecimento da rede dos serviços existentes, o que pode levar à uma carência dos modos compartilhados em saúde ${ }^{13}$.

Deste modo, os profissionais participantes deste estudo observam a necessidade dos serviços que atuam na comunidade como as escolas e as equipes de ESF conhecerem a RAPS direcionada ao cuidado do adolescente usuário de crack e outras drogas, visto que estes encontram-se mais próximos da população e possuem maior potencial para identificar, orientar e encaminhar ao tratamento.

Eu acho que a informação sobre a rede tem que estar nos bairros, porque o nosso público ele não está aqui no centro da cidade, nosso público está nos bairros, nas vilas, e essa informação tem que ser levada exatamente lá. Nós temos um posto de saúde, nós temos creche, nós temos escola pública nos bairros; é justamente esses locais que abrangem esse nosso público. Então, é lá que essa informação tem que estar. Essa informação estando lá, mais fácil vai ficar de se trabalhar e mais conhecimento as pessoas terão, não irão esperar tanto tempo para buscar socorro (p17).

$\mathrm{Na}$ escola de redução de danos eu me surpreendi muito, porque tinham profissionais que nem se quer sabiam da existência do CAPS AD. Me surpreendi de colegas não saberem o que era o CAPS AD (p16).

Na escola de redução de danos, eu participei também das capacitações dos agentes comunitários de saúde, então a gente apresenta a rede, apresenta os serviços e muita gente diz: "nunca ouvi falar", "não sei o que é", "como é que funciona", "não tenho ideia de como é". Acho que a própria rede de saúde ainda desconhece e não tem bem a compreensão do que é um CAPS 24 horas, para que serve, o que é unidade de acolhimento (p18).

Corroborando com esses achados, estudo que analisou a visão de coordenadores da Estratégia Saúde da Família sobre a conformação da rede de atenção psicossocial apontou que a maioria dos gestores desconhecem os serviços de saúde mental disponíveis para o cuidado de pessoas em sofrimento psíquico, $\mathrm{o}$ que, consequentemente, resulta em práticas de cuidado nada efetivas ${ }^{18}$. 


\section{ARTIGO ORIGINAL}

Assim, o desconhecimento da RAPS constitui-se em entrave à articulação e efetivação do cuidado, e por isso precisa ser superado, seja por meio de qualificação profissional, estabelecimento de um fluxograma organizativo dos serviços, apoio matricial para o trabalho em saúde mental na atenção básica. Isso da-se ao desconhecimento sobre a dinâmica de funcionamento desses serviços é um fator limitante na composição de uma rede de atenção, bem como ao acesso pelos usuários, contribuindo para a manutenção do hospital psiquiátrico como alternativa de atendimento ${ }^{19}$.

\section{(Des)ajustes envolvidos na assistência ao adolescente usuário de crack}

Os profissionais apontam a necessidade de se repensar e ajustar o local para o tratamento do adolescente usuário de crack, pois embora o CAPS $A D$ realize o acolhimento e acompanhamento dos mesmos, sua estrutura não contempla as especificidades e necessidades relativas a essa faixa etária. Por esta razão, apontam que o CAPSi teria melhores condições de ofertar a assistência ao adolescente usuário de crack, uma vez que o seu público alvo abrange os usuários dessa faixa etária (Figura III).

Eu acho que o CAPSi poderia dar conta desses adolescentes, dessas crianças. Mas também não sei se eles teriam pernas para tudo isso. Será que eles teriam estrutura? ( $p 15)$.

Penso que o CAPSi poderia absorver esta demanda e teria que ter mais políticas de prevenção ao uso indevido de drogas para adolescentes (p10).

Eu acredito que o CAPSi seria melhor, no sentido de que estaria mais preparado para atender. Inclusive estar preparado para fazer as atividades com esses adolescentes. $O$ que acontece às vezes, esses adolescentes vêm no CAPS $A D e$ aquelas atividades não contam para o que eles necessitam. Então, acredito que o CAPSi poderia contribuir com isso, porque eles vão estar preparados para trabalhar a faixa etária (p8).

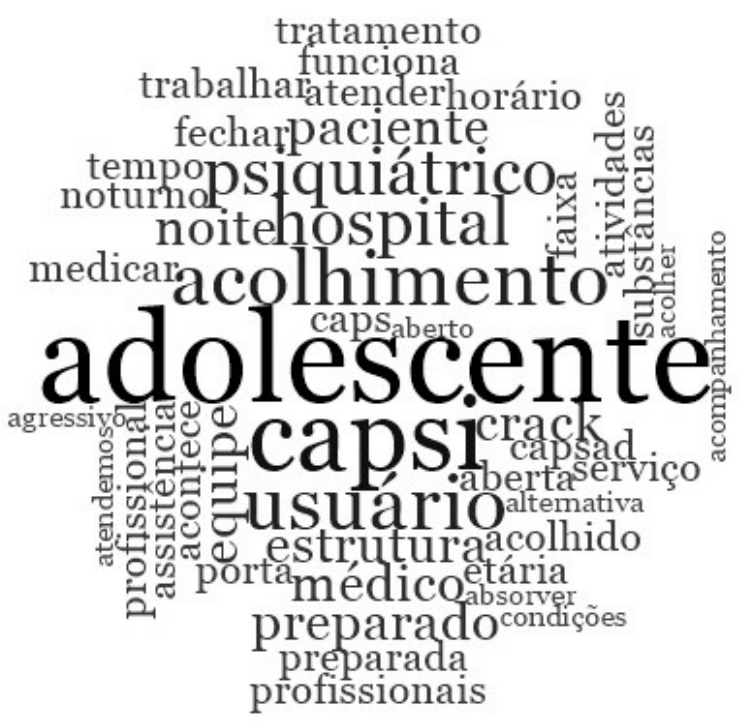

Figura 3 - Nuvem de palavras por frequência da codificação da categoria "Contradições envolvidas na assistência ao adolescente usuário de crack", via QSR NVivo. Pelotas/RS, 2017.

A experiência do município de São Bernardo do Campo - SP mostra que a separação física em unidades distintas entre o CAPS AD adulto e o CAPS $A D$ infanto-juvenil tem sido estratégica para garantir o cuidado adequado às especificidades de cada ciclo de vida, permitindo uma atenção diferenciada e de qualidade. Deve-se reconhecer que o tipo de oficina, de intervenção terapêutica, de projetos de inserção na cidade e diversas outras estratégias para o cuidado são muito variados para essas faixas etárias ${ }^{20}$.

Outro ponto a ser repensado e ajustado é o fato de o hospital psiquiátrico integrar a RAPS como alternativa de atendimento para os casos que envolve comportamento agressivo do adolescente usuário de crack.

Raras são as vezes que a gente encaminha para o hospital psiquiátrico. A situação de crise que não atendemos aqui é porque ia colocar em risco. Como é aberto, então a gente tem que ter essa medida protetiva, digamos assim (p7). 
Já tem um tempo de reforma. Já melhorou bastante, mas a gente está começando. É recente que a gente tem um CAPS AD 24 horas, tem a unidade de acolhimento e a gente ainda tem $o$ hospital psiquiátrico, ele faz parte da rede. Então, ao mesmo tempo em que a gente luta para fechar o manicômio, tem ele como opção. Por exemplo, vamos pensar no adolescente, ele chegou aqui com 16 ou 17 anos, agressivo sob efeitos de substâncias, algemado pelos guardas, então é o Hospital Psiquiátrico que atua para atender este adolescente ( $p 8)$.

Possibilitar a internação de todas as idades em leitos não manicomiais continua sendo um grande desafio, principalmente pelo fato de que o município estudado possui ainda um Hospital Psiquiátrico conveniado ao SUS, sob gestão municipal, que por muito tempo constituiu-se no único recurso de atendimento em saúde mental. $\mathrm{A}$ presença do hospital psiquiátrico na rede constituise em um ponto crítico.

Pesquisadores apontam que o hospital psiquiátrico ainda aparece como ponto de referência no tratamento mesmo em municípios com um sistema de referência e contrarreferência entre a atenção básica e os CAPS I e uma parceria com os outros serviços da rede de saúde mental que ampliam as ações de cuidado ${ }^{13}$.

É imperativo que processos e movimentos da rede de saúde mental infantojuvenil se efetivem com práticas de cuidado norteadas pelo modo psicossocial, no sentido de acolher e cuidar de seus usuários sem encaminhar para o hospital psiquiátrico. Para tanto, faz-se necessário investir em espaços de comunicação e educação permanente das equipes, em melhores infraestrutura dos serviços da rede e em modos de cuidado no território dos usuários da saúde mental ${ }^{11}$.

\section{CONCLUSÕES}

Os resultados apontam que os contrassensos na dinâmica de funcionamento da rede de atenção psicossocial direcionada ao cuidado do adolescente usuário de crack envolvem a (Des)articulação da Rede de Atenção Psicossocial, o (Des)conhecimento dos usuários e profissionais acerca da Rede de Atenção Psicossocial e os (Des)ajustes envolvidos na assistência ao adolescente usuário de crack.

A (Des)articulação da Rede de Atenção Psicossocial aponta que o desenvolvimento de ações intra e intersetoriais, apesar de necessárias, ocorre de forma insipiente no município. Por esta razão, os movimentos de construção desta rede precisam ser fortalecidos, a partir de articulação de ações de prevenção ao uso de drogas, de tratamento e de reinserção social que utilizem os recursos existentes no território da própria comunidade do adolescente.

Dessa forma, potencializa-se o cuidado ampliado em saúde mental, uma vez que busca-se compreender de forma global e contextualizada as necessidades do adolescente, não somente em relação ao uso de crack. Para tanto, faz-se necessário capacitar os profissionais da RAPS para atuar diante das demandas desse público, de forma a superar práticas retrogradas e estigmatizantes que contribuem para a exclusão social e para centralização do cuidado dos usuários de drogas em um único dispositivo de cuidado, o CAPS AD.

Com relação ao (Des)conhecimento da RAPS aponta-se a necessidade da divulgação da mesma à população e aos demais serviços que atuam na comunidade, de forma a ganhar visibilidade e, consequentemente, maior utilização. Assim, evitando-se a procura e utilização do hospital psiquiátrico como alternativa de atendimento.

Deste modo, se não houver mudanças na formação e capacitação profissional, não ocorrerão mudanças na conformação e dinâmica de funcionamento da RAPS, corroborando para que a representatividade social outrora assumida pelo hospital psiquiátrico seja conferida hoje ao CAPS $A D$, ou mesmo contribua para a permanência do hospital psiquiátrico como serviço pertencente a RAPS.

$\mathrm{Na}$ análise dos (Des)ajustes envolvidos na 


\section{ARTIGO ORIGINAL}

assistência ao adolescente usuário de crack evidenciou que o jovem deve ser acolhido e acompanhado por um serviço que atenda não só as necessidades relacionadas ao uso do crack, como também as especificidades relativas a idade. Embora tanto o CAPSad quanto o CAPSi sejam apontados como dispositivos apropriados para o tratamento de adolescentes usuários de substâncias psicoativas, o presente estudo registra a necessidade de ampliação do debate acerca da relevância de um CAPS AD infanto-juvenil como estratégia de cuidado a este público.

Destaca-se como limite do estudo o fato da coleta de dados ter ocorrido em um único serviço que compõe a RAPS, assim retratando os contrassensos por um ponto de vista específico, que não retratam de forma global a realidade da RAPS, uma vez que não foram incluídos outros serviços emblemáticos no cuidado aos adolescentes usuários de crack, álcool e outras drogas como o CAPSi, bem como da área do Conselho Tutelar, Escolar e Justiça.

\section{REFERÊNCIAS}

1. Organización Mundial de la Salud (OMS). Salud Mental y Desarrollo: poniendo a las personas con problemas de salud mental como um grupo vulnerable. Geneva: WHO; 2010.

2. Seleghim MR, Galera SAF, Oliveira MLF. Usuários de crack atendidos em unidade de emergência psiquiátrica: perfil de uma série de casos. Rev. pesqui. cuid. fundam. (Online) [Internet]. 2016 Oct [citado em 201728 ago]; 8 (4): 4907-13, 2016. Disponível em: http://www.seer.unirio.br/index.php/cuida dofundamental/article/view/2961/pdf_1.

3. Nardi FL, Jahn GM, Dell'aglio DD. Perfil de adolescentes em privação de liberdade: eventos estressores, uso de drogas e expectativas de futuro. Psicol. rev. (Belo
Horizonte) [Internet]. 2014 [citado em 2017 04 set]; 20 (1): 116-137. Disponível em: http://pepsic.bvsalud.org/pdf/per/v20n1/v 20n1a.

4. Moretlwe SD, Cowan FM, Busza J, Moore CB, Kelley K, Fairlie F. Providing comprehensive health services for young key populations: needs, barriers and gaps. J Int AIDS Soc [Internet]. $2015 \mathrm{Fev}$ [citado em 201713 dez]; 18 (2): 1-12. Disponível em: https://www.ncbi.nlm.nih.gov/pmc/articles /PMC4344539/pdf/JIAS-18-19833.pdf>.

Acesso em: 13 dez. 2017.

5. Brasil. Portaria $\mathrm{n} 0 \mathbf{3 0 8 8}$, de 23 de dezembro de 2011. Institui a Rede de Atenção Psicossocial para pessoas com sofrimento ou transtorno mental e com necessidades decorrentes do uso de crack, álcool e outras drogas, no âmbito do Sistema Único de Saúde (SUS). Diário Oficial da União 2011; 23 dez.

6. Bernardi AB, Kanan LA. Características dos serviços públicos de saúde mental (Capsi, Capsad, Caps III) do estado de Santa Catarina. Saúde debate [Internet]. 2015 [citado em 201708 jan]; 39 (107): 1105$1116 . \quad$ Disponível em: http://www.scielo.br/pdf/sdeb/v39n107/0 103-1104-sdeb-39-107-01105. pdf.

7. Vasconcelos EM. Cenário econômico, social e psicossocial no Brasil recente, e a crescente difusão do crack: balanço e perspectivas de ação. O Social em Questão [Internet]. 2012 [citado em 201712 jun]; 28: 149-86. Disponível em: http://osocialemquestao.ser.pucrio.br/media/8artigo.pdf.

8. Guizzo BS, Krziminski. CO, Oliveira DLLC. O Software QSR NVIVO 2.0 na análise 
qualitativa de dados: ferramenta para a pesquisa em ciências humanas e da saúde. Rev. gaúch. enferm. [Internet]. 2016 [citado em 201728 jun]; 24 (1): 53-60, 2003. Disponível em: http://www.lume.ufrgs.br/bitstream/handl e/10183/23510/000397047.pdf?sequenc

9. Lima M, Dimenstein M. O apoio matricial em saúde mental: uma ferramenta apoiadora da atenção à crise. Interface comun. saúde educ. [Internet]. 2016 [citado em 201728 jun]; 20 (58): 625-635, 2016. Disponível em: http://www.scielo.br/pdf/icse/v20n58/180 7-5762-icse-1807-576220150389.pdf.

10. Bastos IT, Sarubbi VJ, Oliveira TGP, Delfini PSS, Muylaert CJ, Reis AOA. Identidade do cuidado em Centro de Atenção Psicossocial Infantojuvenil para usuários de álcool e drogas. Rev. Esc. Enferm. USP. [Internet]. 2014 [citado em 201712 jun]; 48 (2): 116$122 . \quad$ Disponivel em: http://www.scielo.br/pdf/reeusp/v48nspe 2/pt_0080-6234-reeusp-48-nspe200116.pdf.

11. Pinho $L B$, Silva $A B$, Siniak $D S$, Folador $B$, Araújo LB. Análise da articulação da rede para o cuidado ao usuário de crack. Rev. baiana enferm. [Internet]. 2017 [citado em 201728 jun]; 31 (1): 1-9. Disponível em: https://portalseer.ufba.br/index.php/enfer magem/article/view/16654/14112.

12. Macedo EOS, Conceição MIG. Atendimento. psicossocial a crianças e adolescentes em situação de violência: o psicólogo e a rede de atenção. Pesqui. prát. psicossociais. [Internet]. 2017 [citado em $201713 \mathrm{dez}$; 12 (1): 129-46. Disponível em: http://pepsic.bvsalud.org/pdf/ppp/v12n1/ 10.pdf.
13. Kantorski LP, Coimbra VCC, Oliveira NA, Nunes CK, Pavani FM, Sperb LCSO. Atenção Psicossocial Infantojuvenil: interfaces com a rede de saúde pelo sistema de referência e contrarreferência. Texto \& Contexto Enfermagem [Internet]. 2017 [citado em 201713 dez]; 26 (3): 1-10. Disponível em: $<$ http:

http://www.scielo.br/pdf/tce/v26n3/01040707-tce-26-03-e1890014.pdf.

14. Varela DSS, Sales IMM, Silva FMD, Monteiro CFS. Rede de saúde no atendimento ao usuário de álcool, crack e outras drogas. Esc. Anna Nery Rev. Enferm. [Internet]. 2016 [citado em 201719 ago]; 20 (2): 296-302. Disponível em: http://www.scielo.br/pdf/ean/v20n2/1414 -8145-ean-20-02-0296. pdf.

15. Xavier RT, Monteiro JK. Tratamento de Pacientes. Usuários de crack e outras drogas nos CAPS AD. Psicol. rev. (Belo Horizonte) [Internet]. 2013 Abr [citado em 201712 jun]; 22 (1): 61-82. Disponível em: <https://revistas.pucsp.br/index.php/psico revista/article/view/16658/12511.

16. Silva $A B$, Pinho $P B$, Olschowskya $A$, Siniaka $D S$, Nunes CK. O cuidado ao usuário de crack: estratégias e práticas de trabalho no território. Rev. gaúch. enferm. [Internet]. 2016 [citado em 201719 ago]; 37 (esp): 2-7. Disponível em: http://www.scielo.br/pdf/rgenf/v37nspe/0 102-6933-rgenf-198314472016esp68447.pdf.

17. Brasil. Portaria no 130 , de 26 de janeiro de 2012. Redefine o Centro de Atenção Psicossocial de Álcool e outras Drogas $24 \mathrm{~h}$ 


\section{ARTIGO ORIGINAL}

(CAPS AD III) e os respectivos incentivos financeiros. Diário Oficial da União 2012; 26 jan.

18. Eslabão AD, Coimbra VCC, Kantorski LP, Pinho LB, Santos EO. Rede de cuidado em saúde mental: visão dos coordenadores da estratégia saúde da família. Rev. gaúch. enferm. [Internet]. 2017 [citado em 2018 08 jan]; 38 (1): 1-8. Disponível em: http://www.scielo.br/pdf/rgenf/v38n1/en_ 0102-6933-rgenf-1983-

144720170160973.pdf.

19. Cortes LF, Terra MG, Pires FB, Heinrich J, Machado KL, Weiller TH, et al. Atenção a usuários de álcool e outras drogas e os limites da composição de redes. Rev. eletrônica enferm. [Internet]. 2014 Jan [citado em 201808 jun]; 16 (1): 84-92, 2014. Disponível em: https://www.fen.ufg.br/fen_revista/v16/n 1/pdf/v16n1a10.pdf.

20. Furtado LAC, Zaparoli C, Chioro A, Robortela, S. Política de Atenção e Prevenção às pessoas em situação de uso abusivo de álcool e outras drogas no município de São Bernardo do Campo - SP. In: Lancetti A, Chioro A, Vargas B, Zaparoli C, Petuco DRS, Castilho EWV, organizadores. Drogas e Cidadania: em Debate. Brasília-DF: Conselho Federal de Psicologia; 2012. p. 53-61. 